University of Nebraska - Lincoln

DigitalCommons@University of Nebraska - Lincoln

Mechanical \& Materials Engineering Faculty

Publications

Mechanical \& Materials Engineering

Department of

2001

\title{
Task Specific Uncertainty in Coordinate Measurement
}

R. G. Wilhelm

University of North Carolina at Charlotte, bob.wilhelm@unl.edu

R. Hocken

University of North Carolina at Charlotte

H. Schwenke

Physikalisch-Technische Bundesanstalt, Braunschweig, Germany

Follow this and additional works at: https://digitalcommons.unl.edu/mechengfacpub

Part of the Mechanics of Materials Commons, Nanoscience and Nanotechnology Commons, Other Engineering Science and Materials Commons, and the Other Mechanical Engineering Commons

Wilhelm, R. G.; Hocken, R.; and Schwenke, H., "Task Specific Uncertainty in Coordinate Measurement" (2001). Mechanical \& Materials Engineering Faculty Publications. 463.

https://digitalcommons.unl.edu/mechengfacpub/463

This Article is brought to you for free and open access by the Mechanical \& Materials Engineering, Department of at DigitalCommons@University of Nebraska - Lincoln. It has been accepted for inclusion in Mechanical \& Materials Engineering Faculty Publications by an authorized administrator of DigitalCommons@University of Nebraska Lincoln. 
Published in Annals of the CIRP 50:2 (2001), pp. 553-563; doi: 10.1016/S0007-8506(07)62995-3

Copyright (C) 2001 CIRP. Published by Elsevier Ltd. Used by permission.

Published online July 6, 2007.

\title{
Task Specific Uncertainty in Coordinate Measurement
}

\author{
R. G. Wilhelm, ${ }^{1}$ R. Hocken, ${ }^{1}$ and H. Schwenke ${ }^{2}$
}

1. Center for Precision Metrology, Department of Mechanical Engineering and Engineering Science, University of North Carolina at Charlotte, Charlotte, North Carolina, USA

2. Laboratorium fuer Koordinatenmessgeraete/CMM Section, Physikalisch-Technische Bundesanstalt (PTB), Braunschweig, Germany

\begin{abstract}
Task specific uncertainty is the measurement uncertainty associated with the measurement of a specific feature using a specific measurement plan. This paper surveys techniques developed to model and estimate task specific uncertainty for coordinate measuring systems, primarily coordinate measuring machines using contacting probes. Sources of uncertainty are also reviewed.
\end{abstract}

Keywords: metrology, uncertainty, modeling

\section{Introduction}

The "task specific uncertainty" in coordinate measurement is the measurement uncertainty that results, computed according to the ISO Guide to the Expression of Uncertainty in Measurement (GUM), when a specific feature is measured using a specific inspection plan. Measurements commonly are of size, position, or form and are made of features dimensioned and toleranced in accordance to international and national standards [1,2]. Even when form alone is measured on a special-purpose machine, uncertainty is difficult to estimate correctly [3]. A correct statement of the uncertainty is becoming increasingly important as more and more companies strive to maintain traceability in accordance with ISO 9000 [4] and begin to comply with the GPS series of standards. In particular, ISO 14253-1 [5] states that a proper uncertainty statement is necessary in order to conclude that products are within or outside of specification. The problem is compounded by the expanded 
outsourcing being used by large corporations and the increasing globalization of the world economy. As the number of production sources used to produce product subsystems increases the necessity for a well-functioning and controlled measurement system expands dramatically. Interchangeability in the global marketplace requires traceability and a thorough estimation and understanding of uncertainty.

\section{Traceability and Definitions}

The necessity for task specific uncertainty is derived from the requirements of ISO 9000 and, more recently, of ISO 17025 [6] for traceability [7]. Traceability is defined as "property of the result of a measurement or the value of a standard whereby it can be related to stated references, usually national or international standards, through an unbroken chain of comparisons all having stated uncertainties." This definition is from the International Vocabulary of Basic and General Terms in Metrology (VIM) [8]. This definition is now also accepted by the National Institute of Standards and Technology, which had previously had no organizational policy in this area (see www.nist.gov/traceability). In order to obtain the correct uncertainties it is currently required to use the techniques outlined in the Guide to the Expression of Uncertainty in Measurement (GUM) [9,10]. In this paper we will, insofar as possible, use the definitions provided in VIM and conform to the guidelines of the GUM.

\section{Sources of Uncertainty}

Uncertainty in coordinate measurement systems (CMS) comes from many sources [11-26]. Estimating the total uncertainty is, however, difficult, due to the fact that coordinate measuring systems are multipurpose measuring instruments whose uncertainties vary with the task being performed, the environment, the operator, the chosen measurement methodologies, etc. For the purposes of this paper we divide CMS uncertainties into five main categories: hardware, workpiece, sampling strategy, fitting and evaluation algorithms [27,28], and extrinsic factors [29]. Each of these sources is separately outlined below.

\subsection{Hardware}

Here we refer to the sources of uncertainty caused by errors inherent to the design of the machine, its scales and geometry, its probing system, its dynamics, and the environment in which it is placed. Included here would be the uncorrected parts of the 21 so-called parametric errors [30-37], random components of these parametric errors [38], uncorrected systematic and apparently random probing errors [39-42], probe changing and probe articulation uncertainties $[43,44]$, probing parameters (such as probe approach rate and distance), errors caused by the dynamics of the machine structure and probe (and servo systems, where applicable) [45-47], and the environmental and machine temperatures [48-58] and vibration (primarily). 


\subsection{Workpiece}

These uncertainties relate to properties of the workpiece and measurement interaction with the workpiece. Part form deviations [59-62], accessibility restrictions, sampling distribution [63,64], clamping effects (distortion), contact mechanics, surface finish (where applicable) $[65,66]$, and elastic deformation due to probing forces constitute the main workpiece-related errors that lead to uncertainties. Uncertainties in coordinate transformations to the part coordinate system are also important [67].

\subsection{Sampling Strategy}

Included here are errors due to inadequate sampling, the interaction of sampling strategy with form error [68], interactions of sampling with complex forms, uncertainty "magnification" due to inadequate datums and even incomplete exactness of transfer of measurement results between calibration artifacts and real parts.

\subsection{Fitting and Evaluation Algorithms}

Algorithm suitability and selection, algorithm interaction with sampling density, and algorithm implementation dominate this error category [27,69].

\subsection{Extrinsic Factors}

This contribution [29] to the uncertainty comes from a number of effects that might, in a gage $\mathrm{R}$ and $\mathrm{R}$ study, be referred to as reproducibility. These variables include apparently minor variations in operator machine interaction (tightening of clamps, failure to wear gloves, etc.) coupled with conditions such as part cleanliness, presence of contaminates, etc. Other operator-selectable options such as filtering might be included here but filtering could also be included under Fitting and Evaluation Algorithms.

These error components that lead to uncertainties are outlined in Figure 1. Other classification schemes that are related to the above are also useful. Trapet et al. [70] have cataloged similar errors and divided them into two categories, those that are customarily accessed by measurements and those that are normally estimated. In the first category they include systematic errors of the probing process, random probing errors, probe changing and probe articulation uncertainties, and systematic and stochastic errors of the CMM geometry. The second category includes uncertainties in the assessments of the systematic errors, long-term changes of the individual systematic errors, temperature influences on these errors, model imperfections, and drift effects. Salsbury [71] uses a categorization scheme that divides the uncertainty into machine components, probe components, part components, and repeatability components. More important, however, than the categorization scheme, is our ability to at least determine all of the uncertainty sources and to include them in an uncertainty evaluation for the measurement at hand. 


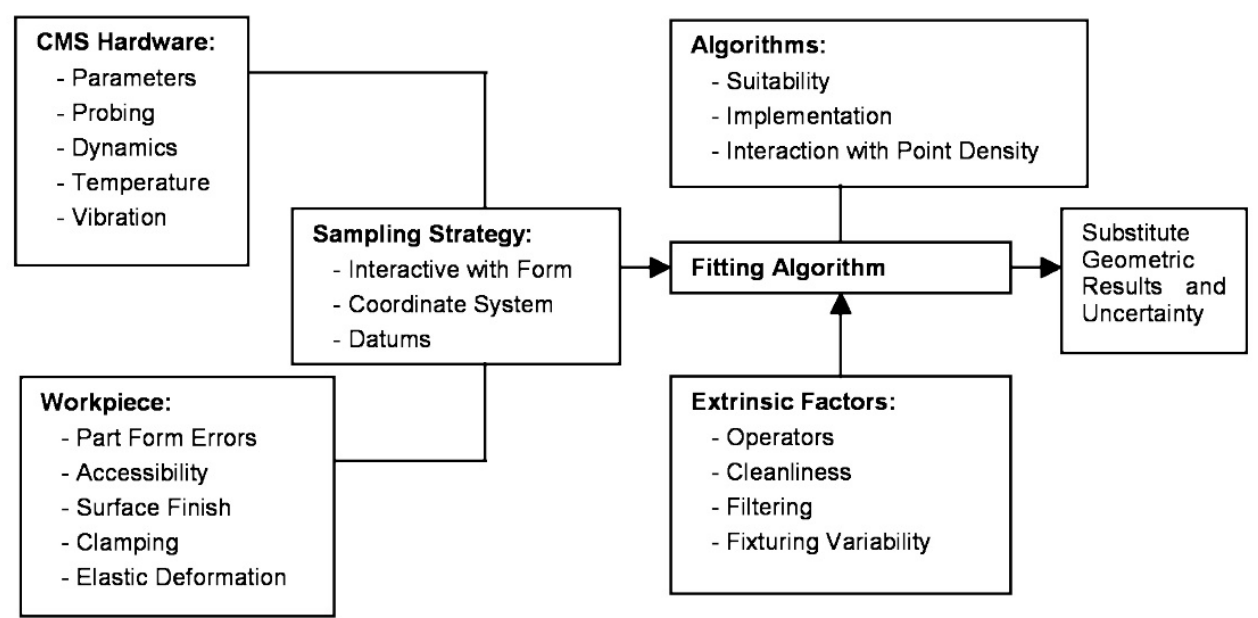

Figure 1. Error components that lead to uncertainties.

\section{Uncertainty Models}

Traditionally metrologists have divided errors into two categories, the so-called systematic errors and random errors. Further, it was traditional to add the uncertainties from random effects in quadrature and then add, arithmetically, an estimation of the systematic effects. This practice is now in the process of being altered due to the publication of the Guide to the Expression of Uncertainty in Measurement (GUM) [9]. The GUM was authorized by seven international standards and metrology organizations, including BIPM, ISO, IEC, IFCC, IUPAC, IUPAP, and OIML, and thus has been uniformly accepted by most national standards laboratories. The approach of the GUM differs from the more classical analysis in that it explicitly realizes that the distinction between systematic and random errors is often ambiguous. It also, in general, makes the assumption that all known biases are corrected before an error analysis is made, although there is active research in this area [72]. Then the uncertainties are divided into two types, Type A uncertainty and Type B uncertainty. Type A uncertainties are those that are evaluated by statistical means, and Type B uncertainties are those that are estimated by "expert" opinion. Estimates of both Type A and Type B uncertainties are added in quadrature and the result multiplied by an appropriate coverage factor to yield an expanded uncertainty. This prescription is extremely general, however, and efforts to realize it for coordinate measuring systems have proliferated. For the purposes of this discussion, we divide those methods into six categories. The first we call sensitivity analysis, the second expert judgment, the third experimental method using calibrated objects, the fourth computer simulation (such as virtual CMMs), the fifth statistical estimations from measurement history [73], and the sixth "hybrid" methods. In the following sections we will discuss the state-of-the-art in each of these categories. 


\subsection{Sensitivity Analysis}

In cases where a clear analytical solution can be formulated for the measurand as a function of measurement parameters, it is relatively easy to follow the prescription of the Guide to the Expression of Uncertainty in Measurement (GUM). As specified in ISO 15530-2 and the GUM, one must first list each uncertainty source, quantify each source according to its magnitude by a standard deviation, determine its sensitivity coefficient and correlation with other uncertainty sources, and add the product of each standard uncertainty by its sensitivity coefficient, in quadrature, and report the combined standard deviation with a coverage factor, typically, of two. This technique is quite useful when a well-defined mathematical model of the measurement process can be ascertained. A good example of this method is given in Phillips et al. [74], where the uncertainty of measuring small circular features is compared to that predicted by a model that includes probe lobing and sampling strategy. Excellent agreement between theory and experiment is obtained.

\subsection{Expert Judgment}

Type B uncertainty determinations, as defined by the Guide to the Expression of Uncertainty in Measurement, typically represent a value judgment based on expert opinion. Significant experience with CMMs, or a specific CMM, combined with feedback on multiple measurements, may provide an expert with the ability to realistically estimate the uncertainty strictly using a Type B method. This technique was primarily used by expert metrologists for decades, as it can be effective for evaluating a single dominant uncertainty source or, in fact, a group of sources, or an entire uncertainty budget. Metrologists in the past actually could estimate the effects of parametric errors entirely without mathematical aids because of a well-developed grasp of the geometrical implications of Abbe offsets, geometric errors, irreproducibility, etc. Expert opinion is perhaps the best technique when other tools and models are not available.

Taking this into account we see some potential for improving and perhaps simplifying the GUM estimation of measurement uncertainties in general, and for CMM task specific measurements in particular, by systemizing expert experiences for quantification, an expert's experience is no other than a-priori knowledge. Such a-priori knowledge is the base of Bayesian statistics [75,76], which is the statistical foundation of the GUM. Unfortunately this is not explicitly mentioned in the GUM and therefore in many cases believed not to be relevant or even in contradiction.

\subsection{Experimental Method Using Calibrated Objects}

The experimental method essentially is a version of the comparator principle that has been used for approximately a century for the measurement of simple artifact standards such as gage blocks (B89.6.2 appendix [77]). The comparator principle has also often been used for the measurement of simple gages [78].

Experimental methods for uncertainty estimation [79] of CMM measurements according to the ISO Technical Specification 15530-3 [80] require a calibrated reference workpiece, which is as similar in its metrological characteristics to the workpiece to be measured as possible. It is based on the statistical evaluation of the measurement errors observed in 
relation to the calibrated value of the reference workpiece. The user must perform a relevant number ( $>20)$ of measurements under the various conditions he might expect while measuring real workpieces. This approach appears to be very simple and straightforward from the view of the user, and attempts to cover intrinsic and extrinsic uncertainty contributors. The method works fine, if the following preconditions are met:

- the calibrated reference workpiece is almost identical to the workpiece to be measured,

- the reference calibration values have a significantly lower uncertainty than the CMS under test, and

- during the experimental investigation, the variation of environmental scenarios and user influences is covered.

The procedure here is to use a CMS to compare a calibrated artifact (master) to the object that is being measured. Conceptually, this may seem simple, but in practice it is fraught with difficulties. Any divergence between the master and the measured part can lead to uncertainties. These would include form deviations (particularly on datum surfaces), differences in thermal expansion coefficients, uncertainties in thermal expansion coefficients and temperature measurements, differences in mounting, operator effects including cleanliness, etc. Even software differences such as coordinate transformations and algorithm sensitivities can contribute. Ideally the master would be identical to the part and measured in the same position on the machine at the same time [12].

One important drawback is the necessity of a reference workpiece, which is calibrated with an uncertainty lower than the CMS under investigation [12]. Due to the general nature of coordinate measuring systems, many masters would be required resulting in a situation similar to the familiar "gage room" where storage, maintenance, cataloging, and calibration of gages is a major expense. In addition, a traceable measurement of the reference workpiece would require a reference method for calibration. One solution to solve this problem is to establish an accredited calibration service for the workpieces based on the simulation method (see section 4.4). This task is currently undertaken in Germany by a consortium of seven private calibration laboratories and PTB. Such a service, combined with the application of the experimental method might make almost any industrial dimensional measurement process traceable.

Some researchers have developed general "gages" that imitate particular part features that are critical. Good illustrative examples are given by Pfeifer [81] and Sammartini $[82,83]$. In this work a "synthetic" gage was manufactured from basic geometric elements (master cylinders) in order to simulate the features encountered in the measurement of bevel gears. Since the gage is made of very high accuracy cylinders which can be assumed to have zero form error, the user can estimate the error that would be produced measuring gear teeth of differing qualities by evaluating the error the machine produces measuring specified "patches" on the cylinders in different machine positions. Similar "gages" or artifacts are being used now to investigate CMS uncertainties when measuring free-form surfaces $[84,85]$ with good results. Although the substitution method can be quite valuable it clearly does not provide a complete solution to the task specific uncertainty problem. 


\subsection{Computer Simulation}

There are many simulation methods that attempt to provide estimates of the task specific uncertainty in coordinate measurement. They all begin with some model of the machine and measurement process which is used as a substitute to the analytical methods of error propagation that work best when a complete closed form mathematical measurement model is possible (or convenient). Various names are given to the methods including the "Virtual CMM" [70,86], "Virtual Instrument" [87], "Simulation by Constraints" [29], the "Expert CMM" [88], or simply Monte Carlo simulation [89].

\section{The Virtual CMM}

The virtual coordinate measuring machine (VCMM) approach estimates an uncertainty statement for a particular measurement task on a particular CMM according to Monte Carlo simulation results. "Basically the virtual CMM performs a point-by-point simulation of measurements, emulating the measurement strategy and the physical behavior of the CMM with the dominating uncertainty contributions disturbing the measurement" [70,90,91]. The simulated measurement should have all the facets of the real measurement that can significantly contribute to the measurement uncertainty. Practical systems include probe qualification and workpiece orientation but in practice may not always cover all contributions. The original developers of the technique considered that there were three basic contributions to uncertainty: known systematic influences, unknown systematic influences, and random influences. They also distinguish between on-line and off-line simulations.

This approach is illustrated in Figure 2 as an extension of a normal CMM measurement. The thick black lines show data flow for a normal CMM measurement while the thick gray lines show the additional data flow that is employed to achieve a VCMM estimate.
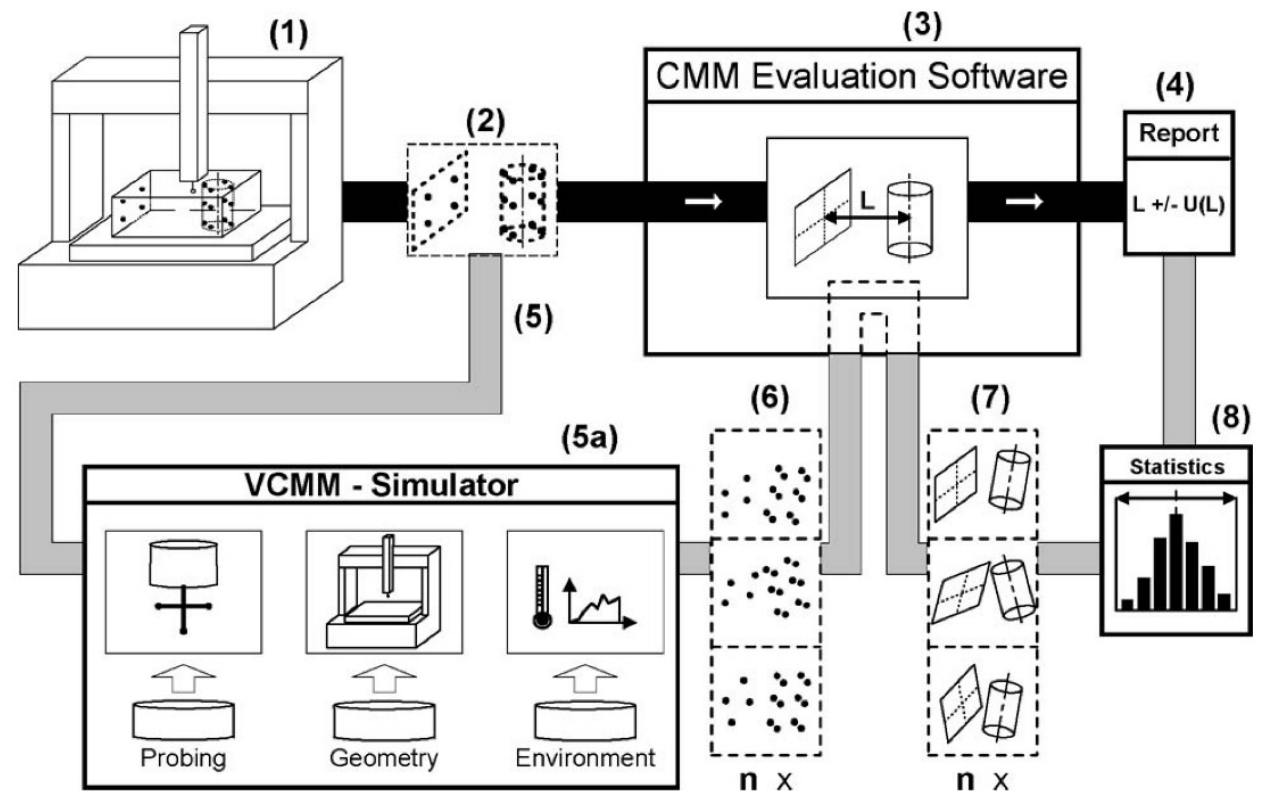

Figure 2. The virtual CMM concept. 
In a normal CMM measurement, a machine with a probe (1) is used to develop a set of points for each feature of interest in the measurement (2). These points are then used with CMM evaluation software to compute substitute geometric elements (3) and derive geometric parameters. These parameters are listed in a measurement report to arrive at an estimate of conformance (4) for each measured feature.

When operating the VCMM, initial input is the set of points (5) to be sampled on a particular feature. These points are specified with respect to the ideal geometry of the design specification. For each sample point on a particular feature, the VCMM simulator (5a) generates a perturbed point that represents an estimate of what a particular CMM would have reported when measuring that commanded point. The perturbed point is generated by modeling variations that may result from part geometry, probing errors, machine motion errors, environmental influences, and other measurement uncertainty contributors. Each error is simulated using a probability density function (PDF) and each point estimate in the simulation is generated by considering all of the PDFs that contribute uncertainty to the measurement result.

For any particular feature, a set of simulated results is generated (6) and then input to typical CMM evaluation software to produce a set of computed substitute geometric elements (7). By evaluating each substitute geometry element, a PDF of the reported parameter can be computed to characterize its uncertainty. This PDF represents the difference between simulated measurements with uncertainty influences present and the same simulated measurement made with no uncertainty influences present. Statistics (8) associated with this PDF can then be used to report the uncertainty of the measurement following the notation of the GUM.

To operate a VCMM, uncertainty contributors must be assessed or estimated for each particular CMM under specific environmental conditions. For each CMM, data files characterizing these uncertainties are used as input for VCMM simulations. These contributors include standard deviations of probing processes, residual errors of CMM motion (scale, straightness, and rotation), permissible temperature gradients, and expansion coefficients. The virtual CMM, as implemented [70], does not include explicitly what we, in this paper, refer to as the interaction of part form error with sampling strategy and extrinsic factors. In the on-line approach the fitting and evaluation algorithms of the machine are tested to some extent.

Typically the uncertainty conditions that are estimated by measurement include systematic errors of the probing process (such as probe lobing), random probing errors, probe changing and probe articulation uncertainty [43,44], and systematic errors of the CMM geometry. A calibrated reference sphere is used to estimate the probing uncertainty. The probing points are distributed at narrow intervals over the sphere with a relatively high density. The measurement is repeated at least once and, using fitting and filtering algorithms, directional error characteristics and random uncertainties from the point data are calculated.

When a probe changer or an articulating probe is used, the sphere is repeatedly measured with the same distribution of probing points but with either different styli or different articulation positions. Again, a standard deviation is calculated and used to represent the uncertainty in this case. If significant position dependence (part position on the CMM) is 
suspected, then either different areas of the CMM have to be examined, or parts must be measured only in the area where probing uncertainties have been assessed. Obviously, even in the same position, only probes or articulating positions that were included in the original tests should be used when measuring.

In order to characterize the errors in the CMM's geometry, parametric errors are used. In the most common instantiation, a calibrated ball plate or hole plate is used [15,92-94], although comparison results of ball plate measurements are not as accurate as could be desired [95]. However, it appears that other techniques, such as direct parametric measurement [30-32], measurement of multiple distances [33,96,97], and self-calibration methods [34,98-100] would also be effective. If a calibrated ball plate is used, measurements are made in several positions and the 18 error functions and three squarenesses are obtained just as in classic parametric calibration. In some cases it is also necessary to measure periodic errors of short wavelength, as is specified in some machine tool standards [101,102] (particularly required when using a ball plate rather than a laser), and these periodic errors are added to the scale errors and straightness errors for each of the three machine axes on a normal three-axis CMM.

Many other uncertainty contributors cannot be easily or economically measured and must be estimated. These additional contributors include uncertainties in the estimation of the parametric and probing errors, long-term changes and temperature influences on these errors, model inaccuracies (such "elastic errors"), and drift effects. In practice these unknown contributors are estimated as either constants or as linear functions related to position, orientation, and/or time.

In order to use the virtual CMM to estimate uncertainties the following is done. First a measuring task and strategy are defined. For each point sampled, systematic and stochastic probing errors and probe change uncertainty (where applicable) are added to the measured value. The point is also perturbed by a drift that is linear with the measurement time, systematic parametric machine errors, and an uncertainty for the parametric errors. Uncertainties are generated using Gaussian generators with specified standard deviations for the various errors. This process is repeated for all $i$ measured points for $\mathrm{N}$ simulated measurement sequences. The results of each sequence are evaluated by the CMM software, and the outputs of all $\mathrm{N}$ measurement sets are compared using normal statistical techniques. For example, the diameter of a circle might be computed $\mathrm{N}$ times and the mean and standard deviation of the diameter reported. Commonly a coverage factor of two would be utilized when reporting the uncertainty.

This method is a variation of Monte Carlo simulation as, for each point measurement a new value for each of the stochastic part of each error is generated from a Gaussian generator with known standard deviation. Early efforts took a long time to run in this fashion so approximations were used to speed up the calculation but this problem will likely resolve itself as computer speed increases.

\section{Simulation by Constraints}

Simulation by constraints is considered by its developers to be a generalization of the "Virtual CMM" described above, a technique that they label full parametric simulation [29]. They call any particular set of parametric errors the "parametric state of the CMM" and 
simulate machines by generating all possible parametric states within known constraints. The concept consists of representing a given parametric state of a machine as a particular point in an infinite dimensional state space. Initially the point could be anywhere in the state space but as knowledge regarding the machine is gained the volume in state space where the particular point could lie is increasingly restricted. Knowledge of the machine state is obtained from measurements of machine performance. If enough information is acquired then it is possible to have a topologically bounded region in the state space in which the machine must lie. They call the set of measurements necessary to bound such a region the "Bounding Measurement Set."

As an illustration of the technique, the method was applied to the parametric errors of a 3-axis measuring machine that, in general, might require several hundred individual measurements to provide a full description. The Bounding Measurement Set was chosen as a subset of the machine characterizing values obtained when testing a machine in accordance with the B89 CMM standard [103]. In particular, the set was based on linear displacement accuracy results for the $\mathrm{X}, \mathrm{Y}$, and $\mathrm{Z}$ axes, the volumetric performance, and the volumetric performance using an offset probe. This set, coupled with the assumptions that straightness errors are a result of angular errors only and ram axis roll is dominated by a linear term, proved to be enough to bound the problem. The procedure for simulating a machine with known B89 performance results was as follows. A random parametric state was selected from the state space and the B89 performance results computed for this set. If the simulated results matched the known results the state was considered a "good virtual state" and the results retained. This procedure was repeated until a large number (several hundred) virtual states were obtained. Once this was done a part could be "measured" on each of the virtual machines and the uncertainty computed as in the virtual machine example above, i.e., using standard statistical techniques on measurement results after analysis by machine software.

The method was verified by measuring a calibrated step gage in 43 different positions and orientations throughout the work zone of a CMM. The B89 tests had been performed on this machine, and it had been simulated by constraints. When the measurement results were compared to the calculated expanded uncertainty from the simulation with a coverage factor of two, $95 \%$ of the measurement errors were contained within the expanded uncertainty calculated according to simulation by constraints.

This work has been expanded into "Error Budgeting" by constraints where ranges of the various parametric errors, such as would be specified when designing and building a machine, are used for the constraints rather than the B89 test results. This method also gave reasonable (but not perfect) results when compared to an existing machine [104].

\section{The Expert CMM}

The Expert CMM project (ECMM) was a collaboration between a national metrology institute and industry [88] and resulted in a modified version of a "Virtual CMM" that has some interesting and unique properties. The authors divide the problem into two conceptual steps: (1) computing the uncertainty of individual point coordinates and (2) propagating this uncertainty through the part program to obtain the final uncertainty in the measurand. 
The method proposed is very general in that the outputs, $y$, of the measuring system are related to the inputs, $x$, through a transfer function, $h(x)$. Nominal measurement points, $x_{0}$, are perturbed with errors from an error simulator using a well-tested Gaussian pseudorandom number generator. The various simulated points are then fed through the CMS software to produce a variety of outputs, $y$, which are evaluated using well-developed statistical techniques to compute the variance-covariance matrix which is all that is required to complete the uncertainty statement.

The system was tested on geometric errors only because they were thought to be complex enough for a thorough trial and have been well known for some years [30]. The parameters for the error model were obtained using a self-calibration technique involving multiple measurements of the length of an artifact in the CMM volume [32]. Testing was done with measurements on a hole plate (two holes, $60 \mathrm{~mm}$ and $20 \mathrm{~mm}$ in diameter, $60 \mathrm{~mm}$ apart). The plate was measured in 100 positions and the length and its uncertainty computed. In $89 \%$ of the cases the calibrated length was within the computed uncertainty with a coverage factor of 2 . None of the other errors shown in Figure 1 were included, so this result can be interpreted as quite successful.

\section{General Monte Carlo Simulation}

Besides the efforts mentioned above, which were focused on CMS, Monte Carlo Simulation has been applied to more general situations where the application to CMSs simply becomes a special case $[89,105-107]$. These authors also explicitly included uncertainties due to uncorrected bias and applied their model to surface roughness measurement using stylus instruments, roundness measurement on form-measuring equipment, as well as the measurement of geometrical features on CMMs. The CMM input quantities were evaluated using methods described previously [108]. In all three cases the uncertainties from the simulation were compared to those obtained from multiple measurements, and the results agreed within theoretical expectations; however, the measurements appear to have been performed at a national metrology institute under controlled conditions. In any event, this effort is expected to lead to software that can be disseminated widely for use as part of a metrology "toolbox." Other authors have also addressed the general use of these types of simulations for uncertainty evaluation in metrology [109].

\subsection{Statistical Estimations from Measurement}

High-volume manufacturing is inherently incredibly efficient from an economic point of view and provides considerable data to allow statistical evaluation of measurement results. In such situations CMMs are often used for routine sampling purposes on parts that are produced in very large numbers. The measurement results from nominally identical features can be compiled to develop a history of measurement results for a particular process and a particular part on a particular (or set of) measuring machine(s) that is(are) being used for process control. Because the measurement results include not only production variability and measurement uncertainty, they perhaps do not reflect true measurement variation, but the variance derived likely overestimates the variation due to the measurement process. Also, it is possible that the results include some measurement bias and its uncertainty. However, if the historical data cover a reasonable time span and the produced 
parts function as intended, it is sufficient to assume that the estimation of uncertainty from this procedure is adequate for the intended end use of the product.

\subsection{Hybrid Methods}

Several researchers have addressed the task specific uncertainty for either specific geometries or in a more general approach that used parts of different methods described above. Some of these approaches are discussed below.

\section{A Mixed Approach}

One of the most complete treatments of CMS uncertainty using a combination of sensitivity analysis and expert opinion was due to Salsbury [71]. He categorized uncertainties into components from the machine, probe, part, and repeatability. Next the relationships between the uncertainty components and the geometric dimensioning and tolerancing "call outs" were qualitatively evaluated, i.e., would a specific error affect a specific tolerance or not. An example is shown in Table 1 for uncertainties due to the machine (his machine errors were nearly identical to those categorized similarly in this paper).

\begin{tabular}{ll} 
Table 1. Relationship between the machine uncertainty component and \\
specific measured features (from Salsbury [71]) \\
\hline Characteristic & Machine \\
\hline Feature of size & Yes \\
Length (not feature of size) & Yes \\
Angle, cone & Possible, more likely for larger surfaces \\
Angle, between features & Yes \\
Flatness & Unlikely, except very large surfaces \\
Straightness & Unlikely, except very large surfaces \\
Circularity (roundness) & Unlikely, except very large surfaces \\
Cylindricity & Unlikely, except very large surfaces \\
Perpendicularity (squareness) & Yes \\
Angularity & Yes \\
Parallelism & Yes \\
Profile of a surface (no datums) & Possible, more likely for larger surfaces \\
Profile of a line (no datums) & Possible, more likely for larger surfaces \\
Profile of a surface (with datums) & Yes \\
Profile of a line (with datums) & Yes \\
Circular runout & Yes \\
Total runout & Yes \\
Position (features of size) & Yes \\
Position (not features of size) & Yes \\
\hline
\end{tabular}

Similar tables were generated for probe and part errors, while repeatability was estimated by examining the normal sources of measurement variation. Application of this technique involves picking a tolerance required on the drawing, determining the uncer- 
tainty values that apply using the tables, estimating a value of the uncertainty using standard acceptance test results, measured repeatabilities, or expert opinion, multiplying by sensitivity coefficients (when required), and adding the results in quadrature. Using this technique clearly requires more operator understanding than the simulation methods mentioned above, but it has the advantage that it clearly spells out uncertainty sources, which should aid in operator education. Excellent results were obtained.

Special Cases

There are several cases in the literature where researchers have examined the uncertainty associated with measuring a specific geometry or due to specific influences. An example for small bores has already been discussed [74]. Because of their overall importance, temperatureinduced uncertainties have also been addressed in some detail [110]. Swyt used the GUM approach, and although he discussed dimensional measurement in general, the results are clearly applicable to CMSs. Another group has used similar concepts to develop a new ISO Draft Technical Report [58], which includes the features of the older American standard [77] as well as uncertainties due to temperature measurement, all in the context of the GUM.

\section{Relationships}

The research concentration on task specific uncertainty is relatively new. Previously many researchers have addressed the issues of machine errors, thermal modeling, and machine testing primarily for the purposes of machine correction and acceptance. These efforts resulted in a series of standards and technical reports. The most widely used among the standards are probably the German VDI/VDE 2617, the ISO 10360 series, and the U.S. B89.4.1.

While developing the methods for estimating task specific uncertainty some researchers took the point of view that the output of the performance tests used for machine acceptance could be utilized as input to their uncertainty estimation approaches. Most prominent of these is the Simulation by Constraints method that uses B89 results [29], but others have used the ISO probing tests [85] and the VDI/VDE length measuring uncertainty tests [71].

The other common point of view is to use the results of the parametric tests [30] coupled with probing tests that are obtained from standards [43] and at least some part of the thermal errors described in standards [58,77]. Although there are supporting documents to standards for the parametric tests performed individually [111] or using artifacts [15], such tests are not part of CMM standards and are only just becoming standardized for machine tools [102]. It is possible that, for any task specific uncertainty method to gain universal acceptance, standardized inputs would be highly desirable if not a requirement.

\section{Conclusions}

Clearly researchers have made good progress in the past decade developing methodologies to estimate task specific uncertainties, particularly for CMMs used in the contact mode acquiring points on an individual basis. There are, however, some gaps that could be addressed to improve these estimates and the ease of their computation. 
First, several of the models require input data that are not commonly measured as part of machine acceptance or interim testing. The utility of these models could be improved by developing standards for machine acceptance and testing that allowed rapid and accurate measurement of the required inputs.

Second, although many of the models were tested in laboratory environments, it is unclear how they would handle the thermal environments common on the factory floor, where temperatures can change by 20 degrees Celsius in a single day [112]. The complexity of thermal effects is well known [50,113-115] and a new ISO working document [58] now exists to help compute their uncertainty, but the methods are not universally understood, even in the case of constant temperatures that differ from $20^{\circ} \mathrm{C}$. Cases where the temperatures are changing are difficult to treat due to the varying time constants of machine components (rams, scales, etc.), parts, fixtures, and probes. These effects are still the subject of active research [53].

Third, none of the models appear (from reading the literature) to successfully address the issues of the interaction of the sampling strategy with possible part form error, which has many ramifications. Even in the case of circles and cylinders $[14,60,116]$ the situation is complex (and difficult to quantify by real measurement due to time and sampling size constraints), but on freeform surfaces it is probably worse [84,85,117-120], and magnification effects due to small datums with form error make matters even worse.

Fourth, there are large classes of coordinate measuring systems that have been only partially addressed in the literature. These include large-volume metrology systems, such as laser trackers, photogrammetry systems, and stereotriangulation [121] as well as visionbased CMMs [122-124], scanning CMMs [45], laser triangulation-based machines, and many others.

Fifth, extrinsic factors are nearly impossible to take into full account without an expert actually examining the measurement situation, conducting the equivalent of a gage $R \& R$ study, and using the data and personal judgement to estimate a Type B uncertainty.

Finally, there are the numerous cases of other CMSs, such as scanning touch probes, laser-based machines, vision machines, laser trackers, and multistation theodolytes that require uncertainty statements [121]. Considerable research remains to be performed.

\section{Bibliography}

[1] ANSI/ASME Y14,5-1994, 1995, Dimensioning and Tolerancing, ANSI, New York.

[2] ISO 1101, 1983, Technical Drawings - Geometrical Tolerancing - Toleranced Characteristics and Symbols - Examples of Indication and Interpretation, International Organization for Standardization, Geneva.

[3] Jusko, O., Salsbury, J. G., Kunzmann, H., 1999, Results of the CIRP-form intercomparison 1996-1998, Annals of the CIRP, 48/1:413-417.

[4] ISO 9000-9004, 2000, Quality Management Systems and supporting documents, ANSI/ASME, New York.

[5] ISO 14253-1, 1998, Geometrical Product Specifications (GPS) - Inspection by measurement of workpieces and measuring instruments - Part 1: Decision rules for proving conformance or nonconformance with specification, International Organization for Standardization, Geneva. 
Wilhelm, Hocken, And SCHWEnke, ANNALS OF THE CIRP 50 (2001)

[6] ISO 17025, 1999, General Requirements for the competence of testing and calibration laboratories, International Organization for Standardization, Geneva.

[7] Hansen, H. N., De Chiffre, L., Savio, E., 2001, “Traceability in coordinate metrology," PRIME 2001 International Conference, pp. 363-368.

[8] ISO, 1993, International Vocabulary of Basic and General Terms in Metrology, International Organization for Standardization, Geneva.

[9] ISO, 1993, Guide to the Expression of Uncertainty in Measurement, International Organization for Standardization, Geneva.

[10] Taylor, B. N., Kuyatt, C. E., 1994, Guidelines for Evaluating and Expressing the Uncertainty of NIST Measurement Results, NIST TN 1297, National Institute of Standards and Technology, Gaithersburg, MD.

[11] Phillips, S. D., Borchardt, B., Caskey, G., 1993, Measurement Uncertainty Considerations for Coordinate Measuring Machines, NISTIR 5170, National Institute of Standards and Technology, Gaithersburg, MD.

[12] Phillips, S. D., 1995, Performance evaluations, in Coordinate Measuring Machines and Systems, Chapter 7, J. Bosch, ed., Marcel Dekker, New York.

[13] Swyt, D. A., 1993, Issues, Concepts, and Standard Techniques in Assessing Accuracy of Coordinate Measuring Machines, NIST TN 1400, National Institute of Standards and Technology, Gaithersburg, MD.

[14] Hocken, R. J., Raja, J., Babu, U., 1993, Sampling Issues in Coordinate Metrology, Mfg. Rev. 6/4:282-294.

[15] Balsamo, A, et al., 1997, Results of the CIRP-Euromet intercomparison of ball plate-based techniques for determining CMM parametric errors, Annals of the CIRP, 46/1:463-467.

[16] Cox, M. G., Forbes, A. B., Peggs, G. N., 2001, Simulation techniques for uncertainty estimation in co-ordinate metrology, NPL Report, National Physical Laboratory, UK.

[17] Forbes, A. B., Harris, P. M., 2000, Simulated instruments and uncertainty estimation, NPL No. CMSC 01/00, National Physical Laboratory, UK.

[18] Knapp, W., 1998, Measurement Uncertainty on Coordinate Measuring Machines, 6th ISMQC IMEKO Symposium, Metrology for Quality Control in Production, Vienna.

[19] Kunzmann, H., Trapet, E., Waldele, F., 1990, A uniform concept for calibration, acceptance test, and periodic inspection of coordinate measuring machines using reference objects, Annals of the CIRP, 39/1:561-565.

[20] Hansen, H. N., De Chiffre, L., and Savio, E., 2001, "Traceability in coordinate metrology," Proc. PRIME 2001, Sestri Levante.

[21] De Chiffre, L., and Hansen H. N., 1997, “Results from an industrial comparison of coordinate measuring machines in Scandinavia," Proc. of the XIV IMEKO World congress, Tampere, Finland, Vol. VIII, p. 22-27.

[22] Hansen, H. N., and De Chiffre, L., 1999, "An industrial comparison of coordinate measuring machines in Scandinavia with focus on uncertainty statements." Precision Engineering 23, p. 185-195.

[23] Meneghello, R., De Chiffre, L., Balsamo, A., 2001, Precision of Coordinate Measurements in Industry: AUDIT ITALIANO, Proc. 2nd euspen Intl. Conf., 346-349, Turin, Italy.

[24] Teeuwsen, J. W. M. C., Soons, J. A., Schellekens, P. H. J., 1989, A General Method for Error Description of CMMs Using Polynomial Fitting Procedures, Annals of the CIRP, 38/1:505-508. 
[25] Hansen H. N., and Trapet E., 1997, "An approach to uncertainty estimation in coordinate metrology." Proc. of the Ninth International Precision Engineering Seminar, Vol. 1, p. 295-297.

[26] Hansen, H. N., 1998, "A database system for uncertainty estimation in coordinate metrology," Proc. of the 6th IMEKO Symposium "Metrology for Quality Control in Production," p. 215-220.

[27] Porta, C., Waldele, F., 1986, Testing of Three Coordinate Measuring Machine Evaluation Algorithms, PTB Report EUR 10909 EN, Directorate-General Science, Research and Development, Commission of the European Communities, Brussels.

[28] Hopp, T. H., 1994, The sensitivity of three-point circle fitting, NISTIR 5501, National Institute of Standards and Technology, Gaithersburg, MD.

[29] Phillips, S. D., et al., 1997, The Calculation of CMM Measurement Uncertainty via the Method of Simulation by Constraints, Am. Soc. for Precision Engineering, 16:443-446.

[30] Hocken, R., Simpson, J., Borchardt, B., Lazar, J., Reeve, C., Stein, P., 1977, Three Dimensional Metrology, Annals of the CIRP, 26/2:403-408.

[31] Zhang, G., et al., 1985, Error compensation of coordinate measuring machines, Annals of the CIRP, 34/1:445-449.

[32] Belforte, G., et al., 1987, Coordinate measuring machines and machine tools self-calibration and error correction, Annals of the CIRP, 36/1:359-363.

[33] Jouy, F., Clement, A., 1986, Theoretical modelisation and experimental identification of the geometrical parameters of coordinate-machines by measuring a multi-directed bar, Annals of the CIRP, 35/1:393-396.

[34] Kruth, J.-P., VanHerck, P., Jonge, L. D., 1994, Self-calibration method and software error correction for three-dimensional coordinate measuring using artifact measuring, Measurement, 14:155-167.

[35] Sartori, S., Zhang, G. X., 1995, Geometric Error Measurement and Compensation of Machines, Annals of the CIRP, 44/2:599.

[36] Schultschik, R., Matthias, E., 1977, The components of the volumetric accuracy, Annals of the CIRP, 26/1:229-233.

[37] Knapp, W., Matthias, E., 1983, Test of the three-dimensional uncertainty of machine tools and measuring machines and its relation to the machine errors, Annals of the CIRP, 32/1:459-462.

[38] Nawara, L., Kowalski, M., 1987, Analysis of the random component of multicoordinate measuring machines and metrological robots position error, Annals of the CIRP, 36/1:373-377.

[39] Estler, W. T., et al., 1996, Error compensation for CMM touch trigger probes, Precision Engineering, 19:85-97.

[40] Estler, W. T., et al., 1997, Practical aspects of touch-trigger probe error compensation, Precision Engineering, 21/1:1-17.

[41] Nawara, L., Kowalski, M., 1985, Influence of the multicoordinate measuring machine head characteristic on circular profiles measurements, Annals of the CIRP, 34/1:449-453.

[42] Miguel, P. C., King, T., Abackerli, A., 1998, A review on methods for probe performance verification, Measurement, 23:15-33.

[43] ISO/TC 213 10360, 1999, Geometrical product specifications (GPS) - Acceptance test and reverification test for coordinate measuring machines (CMM) - Part 5: CMMs using multiple stylus probing systems, International Organization for Standardization, Geneva.

[44] ANSI/ASME B89.4.1-2001, 2001, Methods for the Performance Evaluation of Coordinate Measuring Machines, ASME, New York (in press). 
[45] Pereira, P. H., 2001, Characterization and Compensation of Dynamic Errors of a Scanning Coordinate Measuring Machine, Ph.D. Dissertation, UNC Charlotte, Charlotte, NC.

[46] Weekers, W. G., Schellekens, P. H. J., 1997, Compensation for dynamic errors of coordinate measuring machines, Measurement, 2013: 197-209.

[47] Nawara, L., Kowalski, M., 1984, The investigations on selected dynamical phenomena in the heads of multi-coordinate measuring devices, Annals of the CIRP, 33/1:373-376.

[48] Chakravarthy, B., Cherukuri, H., Wilhelm, R. G., 2001, Prediction of thermal soakout time using analytical models, Accepted by Journal of Precision Engineering.

[49] Balsamo, A., Marques, D., Sartori, S., 1990, A Method for Thermal-deformation Corrections of CMMs, Annals of the CIRP, 39/1:557-561.

[50] Bryan, J., 1990, Thermal Effects, Annals of the CIRP, 39/2:646-656.

[51] Breyer, K. H., Pressel, H. G., 1991, Paving the Way to Thermally Stable Coordinate Measuring Machines, Progress in Precision Engineering, Proc. of IPES 6, Braunschweig, 56-76.

[52] Kruth, J.-P., VanHerck, P., Van den Bergh, C., Schacht, B., 2000, A Parametric Model for Compensation of the Temperature Dependent Geometry of a Coordinate Measuring Machine, Proc. 2nd Intl. Seminar on Increasing Machine Tool Performance, La Baule, France.

[53] Kruth, J.-P., Van den Bergh, C., VanHerck, P., 2001, Correcting Steady-State Temperature Influences on Coordinate Measuring Machines, J. Mfg. Sys., 19/6:365-374.

[54] McClure, E. R., 1967, Significance of Thermal Effects in Manufacturing and Metrology, Annals of the CIRP, 15:61-66.

[55] Weck, M., Herbst, U., 1998, Compensation of Thermal Errors in Machine Tools with Minimum Number of Temperature Probes Based on Neural Network, Proc. of ASME, 64.

[56] Weck, M., McKeown, P. A., Bonse, R., Herbst, U., 1995, Reduction and Compensation of Thermal Errors in Machine Tools, Annals of the CIRP, 44/2:589-597.

[57] Yang, Q. D., Van den Bergh, C., VanHerck, P., Kruth, J.-P., 1999, Study of the Thermal Deformations on a Coordinate Measuring Machine and Compensation via a Neural Network, Intl. J. of Flexible Automation and Integrated Mfg., 7/1\&2:129-147.

[58] ISO/TC213/WG3/N13Xrev.5, DTR 16 015, 2001, Geometrical Product Specifications (GPS) Bias and uncertainty of dimensional measurements due to thermal influences, International Organization for Standardization, Geneva.

[59] Weckenmann, A., et al., 1995, Functionality-oriented evaluation and sampling strategy in coordinate metrology, Precision Engineering, 17:244-252.

[60] Weckenmann, A., Knauer, M., Kunzmann, H., 1998, The influence of measurement strategy on the uncertainty of CMM measurements, Annals of the CIRP, 47/1:451-455.

[61] Woo, T.C., Liang, R., 1993, Dimensional measurement of surfaces and their sampling, Computer Aided Design, 25/4:233-239.

[62] Henke, R.P., et al., 1999, Methods for evaluation of systematic geometric deviations in machined parts and their relationships to process variables, Precision Engineering, 23/4:273-292.

[63] Bourdet, P., Lartigue, C., Leveaux, F., 1993, Effects of data point distribution and mathematical model on finding the best-fit sphere to data, Precision Engineering, 15:150-157.

[64] Choi, W., Kurfess, T., Cagan, J., 1998, Sampling uncertainty in coordinate measurement data analysis, Precision Engineering, 22:153-163.

[65] Anbari, N., Beck, C., Trumpold, H., 1990, The influence of surface roughness in dependence of the probe ball radius with measuring the actual size, Annals of the CIRP, 39/1:577-580. 
[66] DeVries, W. R., Li, C. J., 1985, Algorithms to deconvolve stylus geometry from surface profile measurements, J. of Engrg. for Industry, 107:167-174.

[67] Shen, Y. L., Duffie, N. A., 1991, Uncertainties in the acquisition and utilization of coordinate frames in manufacturing systems, Annals of the CIRP, 40/1:527-530.

[68] Edgeworth, R., Wilhelm, R. G., 1999, Measurement uncertainty due to workpiece error interaction with sampling period, In Machining Impossible Shapes, G. J. Olling, B. K. Choi, R. B. Jerard, Eds., KIuwer Academic Publishers, 196-200.

[69] Hopp, T. H., Levenson, M. S., 1995, Performance measures for geometric fitting in the NIST algorithm testing and evaluation program for coordinate measuring systems, J. Res. of NIST, National Institute of Standards and Technology, Gaithersburg, MD.

[70] Trapet, E., Waldele, F., 1996, The Virtual CMM Concept, In Advanced Mathematical Tools in Metrology II, World Scientific, 238-247.

[71] Salsbury, J. G., 1995, A Simplified Methodology for the Uncertainty Analysis of CMM Measurements, Tech. Paper, Conf. on Precision Metrology/Applying Imaging and Sensoring, Society of Manufacturing Engineers, Indianapolis, IN, IQ95-155:1-22.

[72] Phillips, S. D., Eberhardt, K. R., 1997, Guidelines for expressing the uncertainty of measurement results containing uncorrected bias, J. Res. of the National Institute of Standards and Technology, 102:577-585.

[73] ISO/TC213/WG10 N346 Draft 15530-1 (1998-12-7), 1998, An Overview of Techniques for Determining the Uncertainty in Measurement in Coordinate Metrology, International Organization for Standardization, Geneva.

[74] Phillips, S. D., et al., 1998, The estimation of measurement uncertainty of small circular features measured by coordinate measuring machines, Precision Engineering, 22:87-97.

[75] Press, S. J., 1989, Bayesian statistics: principles, models, and applications, John Wiley, New York, NY.

[76] Estler, W. T., 1999, Measurement as Inference, Annals of the CIRP, 48/2:611-632.

[77] ANSI/ASME B89.6.2-1973(R1979), 1973, Temperature and Humidity Environment for Dimensional Measurement, ASME, New York.

[78] Lingard, P. S., et al., 1991, Length bar and step-gauge calibration using a laser measurement system with a coordinate measuring machine, Annals of the CIRP, 40/1:515-519.

[79] EA-10/05, 1995, Co-ordinate Measuring Machine Calibration, European co-operation for Accreditation, http://www.european-accreditation.org/.

[80] ISO Intl. Tech. Spec. 15530-3, GPS-Coordinate Measuring Machines: Techniques for Evaluation of Uncertainty of Measurement: Use of Calibrated Objects, International Organization for Standardization (to be published soon).

[81] Pfeifer, T., Spur, G., 1994, Task specific gauge for the inspection of coordinate measuring machines, Annals of the CIRP, 43/1:465-468.

[82] Sammartini, M., and De Chiffre, L., 1998, "A task specific gauge for pitch measurement of cylindrical gears," 6th ISMQC (IMEKO).

[83] Sammartini, M., and De Chiffre, L., 2000, "Development and validation of a new reference cylindrical gear for pitch measurement," Precision Engineering, 24:302-309.

[84] Savio, E., De Chiffre, L., 2001, "An Artifact for Traceable Free Form Measurements on Coordinate Measuring Machines," Precision Engineering (in press).

[85] Savio, E., De Chiffre, L., 2001, Performance verification of CMMs for free form measurements, Proc. of 2nd euspen International Conf., Turin, Italy, 362-365. 
Wilhelm, Hocken, And SCHWEnke, ANNALS OF THE CIRP 50 (2001)

[86] Kunzmann, H., Trapet, E., Waldele, F., 1993, Concept for the Traceability of Measurements with Coordinate Measuring Machines, Proc. 7th IPES, Kobe, Japan, 41-52.

[87] Haitjema, H., van Dorp, B., Morel, M., and Schellekens, P. H. J., 2001, “Uncertainty estimation by the concept of virtual instruments," Proc. SPIE 4401 Recent Developments in Traceable Dimensional Measurements, J. E. Decker and N. Brown, Eds., SPIE.

[88] Balsamo, A., et al., 1999, Evaluation of CMM uncertainty through Monte Carlo simulations, Annals of the CIRP, 48/1:425-428.

[89] Schwenke, H., et al., 2000, Assessment of uncertainties in dimensional metrology by Monte Carlo simulation: proposal of a modular and visual software, Annals of the CIRP, 49/1:395-398.

[90] van Dorp, B., Haitjema, H., Delbressine, F., Bergmans, R., Schellekens, P., 2001, “Virtual CMM using Monte Carlo methods based on frequency content of the error signal," Proc. SPIE 4401 Recent Developments in Traceable Dimensional Measurements, J. E. Decker and N. Brown, Eds., SPIE.

[91] van Dorp, B., Delbressine, F., Haitjema, H., and Schellekens, P., 2001, "Calculation of measurement uncertainty for multi-dimensional machines, using the method of surrogate data," Advanced Mathematical and Computational Tools in Metrology V, P. Ciarlini et al., Eds. World Scientific Publishing Co., pp. 344-351.

[92] Trapet, E., Waldele, F., 1991, A Reference Artefact Based Method to Determine the Parametric Error Components of Coordinate Measuring Machines and Machine Tools, Measurement, 9:17-22.

[93] Balsamo, A., et al., 1996, Towards instrument-oriented calibration of CMMs, Annals of the CIRP, 45/1:479-482.

[94] Kunzmann, H., Waldele, F., Salje, E., 1983, On testing coordinate measuring machines (CMM) with kinematic reference standards (KRS), Annals of the CIRP, 32/1:465-468.

[95] Kunzmann, H., Trapet, E., Waldele, F., 1995, Results of international comparison of ball plate measurements in CIRP and WECC, Annals of the CIRP, 44/1:479-483.

[96] Soons, H., Schellekens, P., 1992, On the calibration of CMMs using distance measurements, Proc. of the 4th ISMQC, Tampere, 321-340.

[97] Zhang, G., et al., 1988, A Displacement Method for Machine Geometry Calibration, Annals of the CIRP, 37/1:515-519.

[98] Evans, C. J., Hocken, R. J., Estler, W. T., 1996, Self-Calibration: Reversal, Redundancy, Error Separation, and "Absolute Testing," Annals of the CIRP, 45/2:617-633.

[99] Raugh, M. R., 1985, Absolute two-dimensional submicron metrology for electron beam lithography: a theory of calibration with applications, Precision Engineering, 7/1:3-13.

[100] Coorevits, T., David, J., Bourdet, P., 1991, Elimination of geometrical errors by permutationsApplication to a rotary table, Annals of the CIRP, 40/1:531-534.

[101] ANSI/ASME B5.54-1992, 1992, Methods for the Performance Evaluation of Computer Numerically Controlled Machining Centers, ASME, New York.

[102] ANSI/ASME B5.57-1999, 1999, Methods for the Performance Evaluation of Computer Numerically Controlled Lathes and Turning Centers, ASME, New York.

[103] ANSI/ASME B89.4.1-1995 (formerly B89.1.12M-1990), 1995, Methods for the performance Evaluation of Coordinate Measuring Machines, ASME, New York.

[104] Eisenbies, S., Hocken, R., 2000, Error Budgeting by Constraints, Proc. of ASPE, Scottsdale, AZ.

[105] Haitjema, H., 1992, “Uncertainty propagation in surface plate measurements," Proc. of 4th international symposium on dimensional metrology (ISMQC), Tampere, pp 304-320. 
[106] Haitjema, H., 1996, "Iterative solution of Least-Squares problems applied to Flatness and Grid measurements," Advanced Mathematical and Computational Tools in Metrology II, P. Ciarlini et al., Eds. World Scientific Publishing Co.

[107] Haitjema, H., and Morel, M., 2000, “The concept of a virtual roughness tester," Proceedings X. International Colloquium on Surfaces, M. Dietzch and H. Trumpold, Eds., Shaker Verlag, pp 239-244.

[108] Trapet, E., et al., 1999, Traceability of Coordinate Measuring Machines According to the Method of the Virtual Measuring Machine, PTB-Bericht F-35, Braunschweig, Germany.

[109] Cox, M. G., et al., 2001, Use of Monte Carlo simulation for uncertainty evaluation in metrology, In Advanced Mathematical and Computational Tools in Metrology, P. Ciarlini, et al., Eds., World Scientific, Singapore, 94-106.

[110] Swyt, D. A., 1994, Uncertainties in Dimensional Measurements Made at Nonstandard Temperatures, J. of Res. of the National Institute of Standards and Technology, Gaithersburg, MD, 99:31.

[111] Bechwith, W., Bryan, J., Callaghan, R., Charlton, T., Taylor, B., 1991, Parametric Calibration of Coordinate Measuring Machines, American Society for Mechanical Engineers Tech. Paper, ASME, New York.

[112] Beutel, Dean, 2000, Caterpillar Tractor, Peoria, IL, private communication.

[113] Sartori, S., et al., 1989, A method for the identification and correction of thermal deformations in a three coordinate measuring machine, VDI Berichte, 761.

[114] Bryan, J., McClure, E. R., Pearson, J. W., Brewer, W., 1965, Thermal Effects in Dimensional Metrology, ASME 65, Prod. 13, American Society for Mechanical Engineers, New York.

[115] Bryan, J. B., 1968, International Status of Thermal Error Research, Annals of the CIRP, 16/2:203-215.

[116] Weckenmann, A., Heinrichowski, M., 1985, Problems with software for running coordinate measuring machines, Precision Engineering, 7/2:87-92.

[117] Edgeworth, R., Wilhelm, R. G., 1996, Uncertainty management for CMM probe sampling of complex surfaces, ASME Mfg. Sci. and Engrg., MED-4:511-518.

[118] Edgeworth, R., Wilhelm, R. G., 1999, Adaptive sampling for coordinate metrology, Precision Engineering, 23/3:144-154.

[119] Edgeworth, R., Wilhelm, R. G., 1999, Sampling and measurement uncertainty in coordinate metrology, 14th Annual Mtg. of the ASPE, 389-392.

[120] Edgeworth, R., Wilhelm, R. G., 1999, Sampling approaches for the control of task specific uncertainty in precision metrology, 8th Annual Industrial Engrg. Res. Conf., Section J-I, Phoenix, AZ, Inst. of Industrial Engineers, 8 pages.

[121] Clarke, T. A., et al., 2001, Performance verification for large volume metrology systems, In Laser Metrology and Machine Performance.

[122] Fu, Jiyou, 2000, Illumination Model and Plate Calibration Method for Vision-based Coordinate Measuring Machines, Ph.D. Dissertation, UNC Charlotte, Charlotte, NC.

[123] Hansen, H. N., De Chiffre, L., 1997, A combined optical and mechanical reference artefact for coordinate measuring machines, Annals of the CIRP, 46/1:467-471.

[124] Kim, S. W., McKeown, P. A., 1996, Measurement uncertainty limit of a video probe in coordinate metrology, Annals of the CIRP, 45/1:493-496. 